\title{
Enhancement and depression of tactile and acoustic startle reflexes with variation in background noise level
}

\author{
JAMES R. ISON and JOHN M. RUSSO \\ University of Rochester, Rochester, New York
}

\begin{abstract}
The amplitude of the acoustic startle reflex in the rat is related to the level of broadband background noise by an inverted-U-shaped function. We tested the hypotheses that (1) the initial increment, (2) the subsequent decrement, or (3) the entire function results from a nonspecific noise effect on reflex expression, as would be indicated by a common effect across reflexes elicited by acoustic and nonacoustic stimuli. Responses to electrotactile, airpuff, and acoustic startle stimuli were measured, each in a separate group of rats, in noise levels varying from 60 to $100 \mathrm{~dB}$. A biphasic effect of noise on the acoustic startle reflex was observed, with a modest increase from 60 to 70 or $80 \mathrm{~dB}$, and a decline thereafter. In contrast, electrotactile startle provided a monotonic increase in amplitude to a maximum at 90 or $100 \mathrm{~dB}$, whereas airpuff startle showed a progressive decline in amplitude, with an increase in background level beyond the optimal value at 60 to $70 \mathrm{~dB}$. The latter effects demonstrated an unexpected division between facilitation versus reflex depression within the tactile modality, but subsequent data demonstrated that the effective component of the airpuff was acoustic, rather than tactile, in nature. The overall empirical conclusion is that facilitation by noise is nonspecific, whereas reflex depression that appears at noise levels beyond 70 to $80 \mathrm{~dB}$ is specific to acoustic stimuli. These findings suggest that reflex facilitation results from motor excitation and that reflex depression results from a partial masking of the eliciting stimulus.
\end{abstract}

The amplitude of the acoustic startle reflex in the rat is markedly affected by the presence of homogeneous background noise, an effect first noted by Hoffman and Fleshler (1963). The typical function is biphasic, an initial increase in reflex vigor with a change from low to moderate sound pressure levels giving way to a depression of responding at greater intensities (Ison \& Hammond, 1971). The peak of the nonmonotone function occurs at about $70 \mathrm{~dB}$, but the shape of the function depends in detail on the intensity of the startle stimulus (Davis, 1974), the degree to which the response has been habituated (Cory \& Ison, 1979; Davis, 1974), and the power spectrum of the startle stimulus in relation to the background noise (Gerrard \& Ison, 1990).

The early portion of the inverted-U-shaped function has been hypothesized to reflect an arousal process engaged by the noise, a nonspecific facilitative effect that has been seen in other contexts, including both reflexive and more complex behaviors (e.g., Ison \& Leonard, 1971; MacMillan, Gray, \& Ison, 1973; Sheard, Astrachan, \& Davis,

This research was supported in part by Grants CR-806820 and CR-812129 from the Environmental Protection Agency. We were aided in our statistical analysis by NEI Center Support Grant EY-01319 to the Center for Visual Science and the University of Rochester. John M. Russo is currently at the National Institute for Occupational Safety and Health, 4676 Columbia Parkway, Cincinnati, $\mathrm{OH}$ 45226. Reprint requests may be addressed to James R. Ison, Department of Psychology, Meliora Hall, University of Rochester, Rochester, NY 14627.
1975). The subsequent decremental part of the function lends itself to several explanations. It may be specific to the acoustic startle reflex and may result because the higher noise levels excite the afferent side of the reflex arc and thus partially mask the intense acoustic transient that elicits the startle reflex through one of several modality-specific mechanisms, as Davis (1974) and Gerrard and Ison (in press) suggest. It may otherwise result as a refractory-like consequence of the motor activity observed with intense noise backgrounds (Ison \& Silverstein, 1978), because this activity may reduce the excitability of the motor neurons that also subserve reflex elicitation. This hypothesis was suggested by Ison and Reiter (1980) as part of a one-process arousal explanation of the biphasic inverted $U$. They proposed that initial facilitation may result because increased arousal prepares the motor system by a near-threshold excitation of motor units, which are then more sensitive to the startle stimulus. The later descending portion of the curve may result because more intense noise stimulates these same motor units into action, and thus produces a refractory-like inhibition of reactivity at the time of the eliciting stimulus.

It seemed that these hypotheses could be tested by contrasting the effects of various background noise intensities on startle reflexes elicited by acoustic versus nonacoustic startle stimuli. The standard reflex for these experiments has been the acoustic startle response elicited by a brief intense sound burst. In the main experiment presented here, we contrasted this protocol with two 
(presumably) nonacoustic eliciting stimuli, each of which produced the same sort of startle flinch of approximately the same amplitude. One was a very brief electric shock to the feet, an electrotactile stimulus that was intended to activate various cutaneous receptors and neural processes initiated in the footpads. The second was a puff of air directed at the underside of the rat, which was thought to be effective because it activates cutaneous receptors in the skin associated with hair follicles. If the first phase of the inverted-U-shaped function resulted as a nonspecific arousal effect of the noise, the initial response enhancement should be observed for all three stimulus types. If the second phase of the inverted-Ushaped function resulted as a nonspecific overarousal effect, the terminal response decrement should also be observed for all three stimuli. If, in contrast, one of these two arms of the biphasic function results as a consequence of processes operating uniquely within the auditory system, the effects of the background noise on reflex strength should vary sensibly with the particular modality of the eliciting stimulus.

\section{METHOD}

\section{Subjects}

The subjects were 36 naive male albino rats of the Holtzman strain, with 30 in the main experiment (Experiment 1) and 6 in a subsidiary experiment (Experiment 2). Each subject weighed between 270 and $350 \mathrm{~g}$ and was maintained on an ad-lib food and water schedule and a 12:12-h light:dark cycle.

\section{Apparatus}

The experimental chamber, measuring $8.5 \times 9.0 \times 19.5 \mathrm{~cm}$, was constructed of Plexiglas walls and sides, well perforated for sound transmission, and mounted on compression springs in an aluminum frame. The floor of the cage consisted of two stainless steel plates, $3 \times 19.5 \mathrm{~cm}$ long, which served as the electrodes for the electrotactile shock pulses. The cage was housed in a single-wall sound-attenuating booth (manufactured by the Industrial Acoustics Company, Inc.). The experiments were controlled from solid state equipment housed in an adjacent room.

In Experiment 1, three eliciting stimuli were used. The tonal acoustic startle stimulus was a $10-\mathrm{kHz}$ tone pulse that was $25 \mathrm{msec}$ in duration, including 5-msec rise and decay times, at an intensity of $120 \mathrm{~dB}$ (re: $20 \mathrm{uN} / \mathrm{m}^{2}$ ). It was provided by a Hewlett-Packard oscillator (Model $200 \mathrm{AB}$ ) and was delivered through a University of Rochester electronic switch to a MacIntosh amplifier, then routed through relays synchronized with the electronic switch to a Lansing high-frequency tweeter. The airpuff tactile startle stimulus was a 50 -msec air blast controlled by a pressure gauge set on $15 \mathrm{psi}$ on a compressed air tank. It was gated through a solenoid that was controlled by the timers, then delivered to the floor of the experimental chamber through a six-aperture nozzle designed for an aquarium aerating system. The tip of the nozzle was about $2 \mathrm{~mm}$ below the level of the floor. The electrotactile startle stimulus was provided by a Grass SD-5 stimulator set to deliver single $80-\mathrm{V}, 0.3-\mathrm{msec}$ pulses, at $0.2 \mathrm{~mA}$ (measured in series with a $200-\mathrm{k} \Omega$ resistor) through a Grass constant current unit, to the floor panels of the cage. Preliminary tests had suggested that these three stimuli would yield approximately the same level of startle responding, and that, particularly, the level of shock did not lead to any evident postural changes or apparent escape responses. In Experiment 2, two eliciting stimuli were provided, each using the same airpuff used previously. In one case, the air nozzle was pointed through the floor directly toward the animal in order to excite cutaneous receptors; in the other. it was redirected off to the side of the undercarriage of the cage in order to eliminate its cutaneous properties while preserving its acoustic component. Background noise stimuli were provided by a Grason-Stadler 901-B white-noise generator that fed into a 4-in. cone speaker; they were presented at $60,70,80,90$, and $100 \mathrm{~dB}$ (SPL).

The intensities of all acoustic stimuli were measured from a sample of positions inside the Plexiglas chamber, using a General Radio Co. Type 1561 Precision Sound Level Meter (linear scale), with a Type 1556-B Impact Noise Analyzer for the tonal startle stimuli and the airpuff. An external filter with octave separations was used to measure the spectral composition of the noise background. The peak sound-pressure levels were within a $2-\mathrm{dB}$ range at 1,2 , and $4 \mathrm{kHz}$, fell by $4 \mathrm{~dB}$ at $8 \mathrm{kHz}$, and dropped off at a rate of approximately $10 \mathrm{~dB}$ per octave at frequencies over $8 \mathrm{kHz}$ and under $1 \mathrm{kHz}$. The startle response was measured with the aid of an accelerometer attached to the back of the chamber, which detected the force exerted on the cage by the animal's startle flinch. The output of the accelerometer was amplified and displayed on a storage oscilloscope: stimulus presentation triggered the oscilloscope and the response was measured as peak-to-peak deflection within $100 \mathrm{msec}$ of the eliciting stimulus.

\section{Procedure}

In Experiment 1, variation in background intensity was a withinsubject manipulation. Each animal was placed individually in the cage and given 10 min of acclimatization in the midbackground intensity $(80 \mathrm{~dB})$. The intensity of the background was then changed to the first test background, and five startle stimuli were given $30 \mathrm{sec}$ apart, beginning $24 \mathrm{sec}$ after the intensity shift. Six seconds after the last stimulus in the five-trial series, the second intensity level of the background noise was presented, and the next series of five trials was begun $24 \mathrm{sec}$ later. Each background intensity was presented five times, following the order specified in Latin square designs, separate for each animal; thus, each intensity was presented once in a quasi-random rotation before any was repeated, for a total of $252.5-\mathrm{min}$ noise presentations and a total of 125 startle trials. Startle stimulus modality was a between-subject manipulation. Ten animals were assigned to each startle stimulus condition, receiving either the airpuff, the tone burst, or the electrotactile stimulus to the feet. Mean responding in volts was analyzed in a mixed-design analysis of variance (ANOVA), with noise intensity as the withinsubject factor and startle modality as the between-subject factor, followed by separate analyses. In Experiment 2, the type of eliciting stimulus was a within-subject factor, comparing tactile + sound (with the nozzle directed at the animal) with sound alone (with the airpuff directed away from the animal). The stimuli were presented in 10 blocks of five trials each, with the direction of the airpuff changed between blocks in an irregular order within pairs of blocks. The animals were acclimatized to a 70- $\mathrm{dB}$ background for $10 \mathrm{~min}$; trials then were given at a fixed interval of $30 \mathrm{sec}$ within a block. The experimenter had to enter the experimental room to change the direction of the air nozzle, which necessitated a delay of about $60 \mathrm{sec}$ between blocks.

\section{RESULTS AND DISCUSSION}

Figure 1 depicts the mean startle amplitudes across the five background-noise intensitites and representative within-group standard errors for the three eliciting stimuli used in Experiment 1. Responses to the airpuff and to the tone burst modestly increased with the initial increment in the noise level and then displayed a sharp decline at the higher intensities, the airpuff curve showing an earlier decline. The result for the electrotactile stimulus was 


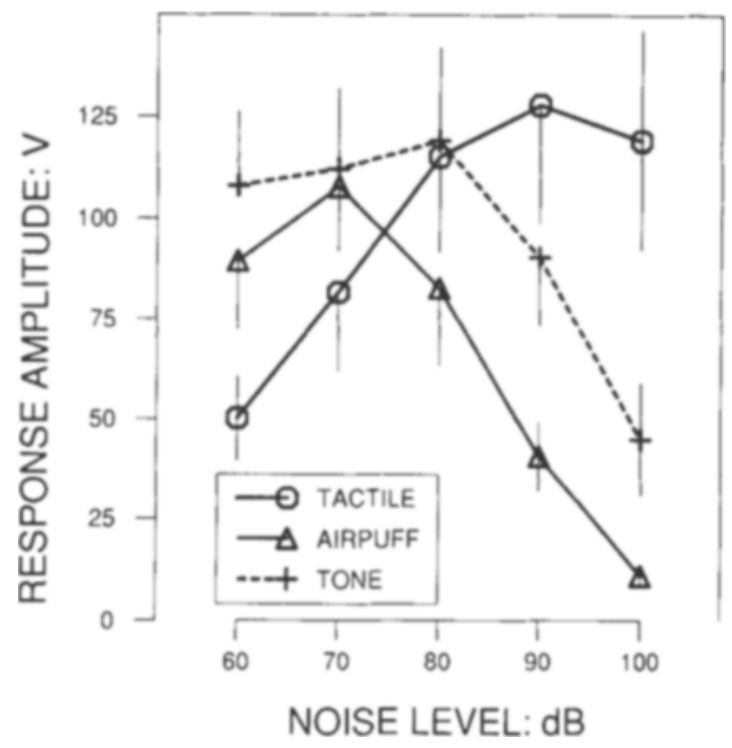

Figure 1. Mean startle reflex amplitudes (in volts) and selected within-group standard errors for three eliciting stimuli-tone burst, airpuff, and electrotactile shock - at each of five background noise levels-60, 70, 80, 90, and $100 \mathrm{~dB}$ (SPL). Note that at $100 \mathrm{~dB}$ the error bar for the airpuff group falls within the symbol.

different from the others: with this stimulus, the response increased with background noise to an asymptote at $90 \mathrm{~dB}$ and did not evidence a significant terminal decline at $100 \mathrm{~dB}$. The ANOVA of these data substantiated the reliability of the visual display of group differences. The analysis produced a main effect for background-noise intensity $[F(4,108)=11.28, p<.01]$ and a reliable interaction of background intensity and modality of the eliciting stimulus $[F(8,108)=13.57, p<.01]$. Modality by itself was not a significant factor in determining response amplitudes $(F=1.14)$. Orthogonal comparisons within this ANOVA indicate (1) that there was an overall linear effect of intensity $(p<.05)$ and that the linear effect interacted with stimulus modality $(p<.01)$, (2) that an overall quadratic effect was significant $(p<.01)$ and did not interact with modality $(F=0.22)$, and (3) that a cubic effect interacted with modality $(F<0.01)$. A secondary analysis compared only the airpuff group against the tone group: the overall linear, quadratic, and cubic components of the intensity function were significant $(p<.01)$, and the cubic component interacted with startle modality $(p<.01)$.

Other analyses showed that the intensity variable was significant in each of the three groups $(p<.01)$, but clearly the expression of the effect was different in each group. To show these separate effects, we made further comparisons of the noise level at which the subjects had their peak responding. In the electrotactile group, these peaks were predominantly at the high end of the noise dimension: 3 animals had their peak at $100 \mathrm{~dB}, 5$ at $90 \mathrm{~dB}$, and 2 at $80 \mathrm{~dB}$. In the airpuff group, the peaks were predominantly at the low end of the noise dimen- sion: 4 had their peak at $60 \mathrm{~dB}, 4$ at $70 \mathrm{~dB}$, and 2 at $80 \mathrm{~dB}$. In the tone group, the peaks were predominantly at intermediate noise levels: 5 had their peak at $80 \mathrm{~dB}$, 3 at $70 \mathrm{~dB}$, and 1 each at 60 and $90 \mathrm{~dB}$. A chi-square test was performed on these data collapsed into three categories of background noise: low $(60$ and $70 \mathrm{~dB})$, midrange $(80 \mathrm{~dB})$, and high $(90$ and $100 \mathrm{~dB})$. The analysis showed that these distributions of the location of maximal responding were reliably different across the three groups $\left[\chi^{2}(4)=22.67, p<.01\right]$. In addition, dependent $t$ tests were used to test whether the increases and the decreases within each group were significant. (It should be noted that these tests used the within-subject standard errors, which were considerably smaller than the betweensubject errors shown in Figure 1 because individual differences in response size were highly correlated across noise levels: for a typical example, within the tone group, the correlation between the responses at 60 and $80 \mathrm{~dB}$ was 0.98.) The $t$ tests that were significant beyond $p<.01$ showed that, (1) in the tone group, the increase from the response at $60 \mathrm{~dB}$ to the peak response at 70 or $80 \mathrm{~dB}$ was reliable; (2) in the same group, the subsequent decline in response amplitudes from the peak to the smaller amplitudes obtained in the presence of $100 \mathrm{~dB}$ was reliable; (3) in the airpuff group, the decline from peak responding at 60 or $70 \mathrm{~dB}$ to the smaller response at $100 \mathrm{~dB}$ was reliable; and (4) in the electrotactile group, the increase in responding from $60 \mathrm{~dB}$ to the larger response at $100 \mathrm{~dB}$ was reliable.

Thus, in summary, the chi-square test showed that the distributions of peak responding differed across the three groups, and the $t$ tests showed that increased noise levels led to an increase in responding in the electrotactile group, to a decrease in responding in the airpuff group, and to a biphasic function in which an initial increase was followed by a decrement in responding in the tone group.

The performance of the acoustic startle group was typical of the results of comparable experiments reported in the literature, showing an inverted-U-shaped relationship between response amplitudes and the level of broadspectrum background noise. The ascending portion of the curve was not as evident as is shown in some prior reports, but this might be expected because here the lowest noise value was $60 \mathrm{~dB}$, whereas in other research, noise levels as low as $30 \mathrm{~dB}$ have been used (e.g., Ison \& Silverstein, 1978). The empirical goal of Experiment 1 was to determine whether this prototypic biphasic curve was unique to the case of reflex elicitation by an acoustic stimulus, or whether it also could be obtained with stimuli in another modality. This question has clear implications for the theoretical meaning of this phenomenon. However, the disparate performance of the two "nonacoustic" startle groups badly complicates this analytical purpose, because it suggests that the suggested dichotomy between acoustic versus nonacoustic elicitation is not critical for understanding the behavior: obviously, some sense must be made of this disparity before the original question can be pursued. 
The decision to use the airpuff procedure was based on the belief that the puff of air would excite cutaneous receptors on the belly of the rat and provide a "natural" reflexogenic event that would add generality to the findings obtained with electric shock. Although electric shock is silent and is known to elicit a startle-like flinch response (Hoffman, Fleshler, \& Abplanalp, 1964) that could be modified by preliminary stimuli (Ash, Parisi, \& Ison, 1978), it is artificial and excites cutaneous receptors and other afferent processes in the footpads of the rat in a nonphysiological fashion. Unfortunately, the seemingly more natural airpuff had a significant acoustic component, a combination of the click of the solenoid closing and a hissing noise. Together these were measured at $115 \mathrm{~dB}$ from a spot $7 \mathrm{~cm}$ away from the nozzle (which would be about the distance of the rat's head from the center of the box). Although it was not possible to examine the spectral content of the airpuff with our filtering equipment (which could only be used with prolonged stimuli), this noise level is sufficient to elicit an acoustic startle reaction across a wide range of frequencies (Fleshler, 1965).

A plausible ad hoc hypothesis that would be consistent with the data in Experiment 1 and with the measurements of the airpuff's noise level is that the airpuff did not function as a second cutaneous stimulus, but rather as a second acoustic stimulus. Experiment 2 was designed to test this hypothesis. If the acoustic component of the airpuff was sufficient to elicit a reflexive flinch response, then the animal should respond to the puff when the force of the air was diverted to the underside of the cage floor. Changing the position of the nozzle also reduced the intensity of the acoustic stimulus (by $8 \mathrm{~dB}$ ), so any modest difference between the two conditions could be attributed to this change in the acoustic input rather than to the loss of the cutaneous component of the stimulus. However, if the cutaneous component of the airpuff were primarily responsible for reflex elicitation in this condition, the response should be largely eliminated by changing the position of the air nozzle. The data obtained in this supplementary experiment reveal that the unique cutaneous component of the airpuff was relatively weak in comparison with the acoustic component. When the nozzle was pointed into the cage, the mean response was $12.73 \mathrm{~V}$, whereas, when the nozzle was deflected to the side the cage, the mean response was $9.64 \mathrm{~V}$ : A dependent $t$ test on these two means gave a value of 0.77 . No animal had a significant difference in responding favoring one nozzle position over the other, and the response of two animals was slightly greater when the nozzle was in the shifted position. These data indicate that the response to the airpuff stimulus in these experiments should be interpreted as being a combination of a dominating acoustic reaction and, at best, a weaker cutaneous reaction. These results suggest that only the electrotactile stimulus is relevant to the question of how background-noise levels affect the nonacoustic startle reflex.

With this conclusion in mind, the fact that the descending part of the inverted-U-shaped function was confined to the startle reflex elicited by the (two) acoustic stimuli supports the argument that the processes responsible for reflex diminution in these groups are located in the afferent arm of the acoustic startle reflex arc. The greater depressive effect of the noise on the airpuff group, relative to the tone group, is consistent with this analysis because the acoustic component of the airpuff was weaker than was the tonal stimulus in the explicit acoustic condition, and it may be anticipated that masking will be more evident with a weaker stimulus (Davis, 1974). A maskinglike effect could be achieved at several levels of the auditory systems in ways that are not incompatible with each other. For example, it is likely that high noise levels excite the intratympanic reflex in the rat (see Kiang \& Peake, 1988 , for a recent review of this phenomenon). The reflex is a graded response of the stapedial muscle to prolonged and intense noise levels. The reflex is hypothesized to serve a protective function (at least in part), because, when the muscle contracts, the ossicular chain that transmits vibratory stimuli through the middle ear stiffens and thus diminishes acoustic input to the inner ear. It is also likely that the prolonged and intense noise backgrounds overdrive both receptive elements in the inner ear and more central afferent neurons. This may reduce the sensitivity to the auditory system to further stimulation or partially mask the additional input of the eliciting stimulus.

The absence of a depressive effect in the shock condition is consistent with the hypothesis that reflex depression results from some sort of a change in responsivity within the auditory system. The intratympanic reflex cannot affect tactile input, nor can this stimulus be masked by the noise, since the two modalities excite different receptors and are processed through a different afferent system. It is possible that the motor overarousal hypothesis suggested by Ison and Reiter (1980) may play some additional role in determining reflex amplitudes at the very highest noise levels: It may be responsible, for example, for limiting the growth of the facilitative effect of noise beyond $90 \mathrm{~dB}$ for the electrotactile stimulus, but it cannot account for the early appearance of depression obtained with acoustic stimuli.

The potentiation of the electrotactile reflex by noise is clearly consistent with the understanding that noise produces a nonspecific arousal process that energizes motor output. These data do not absolutely rule out the contrary hypothesis that response potentiation occurs because a modest level of background noise has a tonic facilitatory effect on the auditory system, but they do show that this hypothesis is neither necessary nor sufficient to explain the present data.

In summary, the present data argue for three major points. The first is primarily a methodological caution: We note that the airpuff stimulus with which we had intended to elicit a tactile startle reflex had a major acoustic component, and that this auditory component was responsible for a significant share of the elicited response. No doubt the degree to which noise contaminates an airpuff stimulus is determined by laboratory-specific details 
having to do with the type of solenoid used, as well as the speed of the air stream, and these findings should not be generalized too far. Nonetheless, other investigators who wish to contrast acoustic and nonacoustic startle behaviors should fractionate their stimuli, perhaps along the lines of Experiment 2, so as to be sure that behavioral differences can be properly attributed to modality differences rather than to differences within the acoustic dimension. The second and third conclusions are more oriented towards a theoretical account of these effects. The ascending arm of the biphasic function that links startle behavior to background-noise level results from a nonspecific facilitatory process and seems reasonably attributed to an arousal mechanism that influences motor expression. In contrast, the descending arm of this same function results in large measure from a specific depressive effect on the acoustic startle reflex, and this seems reasonably attributed to a masking process that is confined to the sensory limb of the reflex arc.

\section{REFERENCES}

ASH, B. L., PARISI, T., \& Ison, J. R. (1978). Modification of acoustic and nociceptive reflexes in the rat by visual stimulation. Animal Learning \& Behavior, 6, 111-114.

Cory, R. N., \& Ison, J. R. (1979). Persistent effect of noise on the acoustic startle reflex in the rat. Animal Learning \& Behavior, 7, 367-371.

DAvIS, M. (1974). Signal-to-noise ratio as a predictor of startle amplitude and habituation in the rat. Journal of Comparative \& Physiological Psychology, 86, 812-825.
FLESHLER, M. (1965). Adequate acoustic stimulus for startle reaction in the rat. Journal of Comparative \& Physiological Psychology, 60, 200-207.

GERRARD, R. L., \& Ison, J. R. (1990). Spectral frequency and the modulation of the acoustic startle reflex by background noise. Journal of Experimental Psychology: Animal Behavior Processes, 16, 106-112.

HoffMan, H. S., \& Fleshler, M. (1963). Startle reaction: Modification by background acoustic stimulation. Science, 141, 138-140.

Hoffman, H. S., Fleshler, M., Abplanalp, P. L. (1964). Startle reaction to electric shock in the rat. Journal of Comparative \& Physiological Psychology, 58, 132-139.

Ison, J. R., Hammond, G. R. (1971). Modification of the startle reflex in the rat by changes in the auditory and visual environments. Journal of Comparative \& Physiological Psychology, 75, 435-452.

IsON, J. R., LEONARD, D. W. (1971). Effects of auditory stimuli on the amplitude of the nictitating membrane of the rabbit (Oryctolagus cuniculus). Joumal of Physiological \& Comparative Psychology, 75, 157-164.

IsON, J. R., \& ReITER, L. A. (1980). Reflex inhibition and reflex strength. Physiological Psychology, 8, 345-350.

Ison, J. R., \& SilversteIN, L. (1978). Acoustic startle reactions, activity, and background noise intensity, before and after lesions of medial cortex in the rat. Physiological Psychology, 6, 245-248.

KIANG, N. Y. S., \& Peake, W. T. (1988). Physics and physiology of hearing. In R. C. Atkinson, R. J. Herrnstein, G. Lindzey, \& R. D. Luce (Eds.), Stevens Handbook of Experimental Psychology (2nd ed., Vol. 1, pp. 277-326). New York: Wiley.

MacMillan, A. St. C., Gray, J. A., \& Ison, J. R. (1973). An apparent new instance of stimulus intensity dynamism during discrimination of duration of repeating auditory stimuli. Quarerly Journal of Experimental Psychology, 25, 62-70.

Sheard, M. H., Astrachan, D., \& Davis, M. (1975). Effect of noise on shock-elicited fighting in rats. Nature, 257, 43-44.

(Manuscript received August 14, 1989; revision accepted for publication November $3,1989$. ) 